\title{
The Audit in the Clinical Trial
}

\author{
Michel Bourin* \\ Neurobiology of anxiety and mood disorders, University of Nantes, France
}

Received: February 27, 2018; Accepted: April 16, 2018; Published: April 27, 2018

*Corresponding author: Michel Bourin, Neurobiology of anxiety and mood disorders, University of Nantes, 98, rue Joseph Blanchart, 44100 Nantes, France,

E-mail: michel.bourin@univ-nantes.fr

\begin{abstract}
Independent and methodological review of clinical research activities and documents to determine whether the activities related to the establishment and monitoring of this clinical research were conducted, if the data were collected, analyzed and reported in compliance with the protocol, standardized operating procedures. System audits should be of a reasonable size and easily manageable with the resources provided (can be managed). Audits must be organized and conducted in such a way that they generate in acceptable results. Most importantly, do not downgrade your audit program, leave windows for nonprogrammed audits.
\end{abstract}

Key words: Audit; Clinical Trial; Quality Evaluation;

\section{Introduction}

The term "audit," long used to refer to the checking of financial accounts, has been applied to clinical trials. The role of the auditor as a person outside the system is to testify that the information contained in reports is credible, i.e. that it has been generated, collected and processed according to the rules of the art. This supposes that the rules (procedures) have been determined and that they respect the laws and regulations.The auditor is a witness within the process of quality evaluation [1].

The quality of a trial depends in part on the assurance and internal control provided by the promoter. Quality assurance is intended to define and establish the conditions or rules for conducting a trial. These procedures are especially concerned with what is to be done, how it is to be done and the way records are to be kept. There are particular procedures for each stage in the process of performing a clinical trial. The investigators need to know the procedures for recording observations in data sheets and binders and managing serious events, treatment units, etc. Quality control ensures that these procedures are carried out. The audit testifies to their application. It is essentially an administrative process which assures the promoter and state supervisory agencies that the rules have been respected and that the data are thus credible.

\section{Setting up the audit}

\section{a) The Objective}

As the audit is intended to verify what has taken place, its form depends on the mission of the auditor, i.e. on the objective in question. It can be a system audit of the promoter or an aspect of the trial such as the galenic files of the treatment units, the maintenance of records, the biometry, or the work of clinical research teams. Or the audit can be conducted on site by a systematic assessment of the work of the investigators, or by random sampling, or, in case of suspected fraud, by the service company in charge of the trial. Laboratories conducting biological or complementary examinations can also be audited. Thus, the choice is large and must necessarily be limited. System audits are most often performed regularly to assess the general situation without any specific reference to a clinical trial. However, onsite audits relate directly to a clinical trial and are conducted especially to improve the credibility and thus the admissibility of the trial by the state supervisory agencies [2].

\section{b) Principle of the Audit}

The auditor should verify that the working rules envisaged by the firm have been applied. These are expressed through procedures which constitute sets of directions relative to all the actions undertaken to reach a goal indicated by the type of procedure in question. Thus, a procedure for record-keeping will indicate who is responsible, who applies the procedure, how the documents should be filed, etc. In addition, it determines the rules for consulting the records and borrowing and duplicating them. Any participant should be able to store records, look for and find a document simply by following the procedure without consulting a succession of persons to obtain explanations.The auditor, in this case, will verify the existence of the procedure, determine that records have been filed as indicated in the procedure and, if necessary, test the procedure on an outsider to be certain that it is valid.

The auditor can be asked to evaluate the relevance of the procedure to its objective. Naturally, procedures are not intended to gratify the ego of their designers but to assure those responsible for an action that it can be carried out effectively. Nevertheless, procedures need to be in accordance with the laws of the country and with good clinical practices. If requested, the auditor can give his opinion about the relevance of the procedures and their adequacy in terms of the intended objective. In fact, the procedures are not a guarantee of quality but the description of working practices. Procedures may be similar from one firm to another but not identical because of differences in the environment and working practices [3]. 


\section{c) Audit Rules}

An audit cannot be performed secretly as it is an important act for firms. The question or questions raised by an audit must always be indicated in advance since they determine how it will be carried out:

1. The firm must advise all the teams concerned of the decision to perform an audit by an individual or a team.

2. The auditor must clearly understand the objective of his mission, i.e. what must be audited and why. He makes sure that the persons involved are clearly aware of the purpose of the audit.

3. The auditor familiarizes himself with all the information required for his work.

4. With the assistance of the sponsor, the auditor draws up an operating plan and an evaluation scale.

5. In practice, the auditor conforms to the accepted plan and evaluates compliance with the working rules (procedures).

6. The auditor keeps records of his meetings, draws up a general report of what he has observed and finally gives his opinion about compliance with the procedures he has investigated and their admissibility by state supervisory agencies. The auditor expresses a clear judgment but does not make a decision. His judgment should be factual since it may be contested by others. In addition to the audit itself, the firm can request advice and opinions about the effective operation of a department, although these observations must not be included in the report.

\section{The system audit}

The system audit is both easy and difficult. It is generally easy since most of the persons involved are present on the site, but it is difficult as well since all of the actions of the teams are not codified (unwritten practices are the rule). The persons in charge of quality assurance and control are overworked and generally do not concentrate on the essential elements expressed in official texts. Finally, the units or departments in a firm are not isolated, even if there is little communication among them (as is generally the case). Interdepartmental interfaces are vital and yet difficult to manage because of the overlapping of responsibility. System audits are generally intended to accomplish the following:

1. To check that all actions have been performed in accordance with the laws and regulations and that the administrative documents are complete.

2. To ensure that no document essential to the operation of a department is lost and that it is used, checked and filed in an acceptable manner.

3. To determine that it is impossible in the operation of the departments to modify an essential piece of information without leaving an explicit indication. Consequently, the auditor should verify that no fraud is possible or, if detected, that it is reported to the highest authorities concerned and given special attention.
It is not the role of the auditor to make a judgment about the organization of departments, which relates to another type of mission. However, it is his duty to indicate the inadequacy of procedures or their possible absence. The auditor should generally comment on the disparity between the description of the procedure and the true state of operations. In fact, procedures should reflect reality and may thus be modified. For this reason, the system audit is often used by managers to improve the operations of departments and check their adequacy in terms of the requirements of authorities, in the event of an official inspection of a particular trial.

\section{On-site audit}

The on-site audit is performed to check the work of the investigators and often of the monitoring team in a particular trial. This audit is intended to provide the trial with an attestation of conformity and thus make it more credible in the eyes of the authorities. The auditor makes a judgment about the compliance of the teams with the rules initially set forth. The audit is announced and described during the meetings with investigators before the trial.When the plan of the audit is developed, the procedure will be described, indicating in particular what will be verified on-site. This procedure should be sent to all investigators before the audit takes place so that they can become familiar with the rules to be followed, the number of sites audited and the actual date of the audit.

The following elements define the plan of action of the audit:

- The protocol and the data sheets and binders;

- The procedures for starting up the trial, the meetings of investigators, the pre-investigation visits, the setting up of the trial, the follow-up and the closing of the centers, as well as the procedures for administrative management of the trial within the firm;

- The list of centers;

- For each center audited, the observations are already noted as well as the reports of visits.

The investigator is advised of the auditor's visit, which occurs independently of that of the monitoring team. The auditor checks all the data required and the way in which they were obtained. In particular, he ensures that the patients included in the trial exist, have given their informed consent and have been seen at the times indicated in the binders.He determines whether the investigator and his team are performing the clinical trial according to the protocol and the established rules of evaluation. If there are procedures specific to the trial, the auditor ensures that they are understood and followed.

After the visit, the auditor prepares a report which is sent to the promotor and, if previously agreed, to the investigator. In his report, the auditor indicates all the failings noted and evaluates patient by patient, and then in terms of the center, the degree of credibility that can be accorded to the trial data. After the audits of all the sites have been performed, the auditor writes a general report indicating his opinion on the credibility of the trial. 


\section{Conclusion}

The audit provides an independent evaluation [5] of the activity of one or more of the trial teams relative to the established rules and generally on the basis of administrative documents. In this respect, there is no direct relation between the quality of a trial and the audit, although the absence of administrative conformity is an indication of poor quality. However, it is possible to extend the notion of quality to the entire trial by taking into account all the elements involved, and notably the way in which the trial data were obtained.

\section{References}

1. Chan AW, Tetzlaff JM, Gøtzsche PC, Altman DG, Mann H, Berlin JA, et al. SPIRIT 2013 explanation and elaboration: guidance for protocols of clinical trials. BMJ. 2013;346:7586. doi: 10.1136/bmj.e7586
2. Chaturvedi M, Gogtay NJ, Thatte UM. Do clinical trials conducted in India match its healthcare needs? An audit of the Clinical Trials Registry of India. Perspect Clin Res. 2017;8(4):172-175.

3. Rostami R, Nahm M, Pieper CF. What can we learn from a decade of database audits? The Duke Clinical Research Institute experience, 1997--2006. Clin Trials. 2009;6(2):141-150.

4. Bello S, Wei M, Hilden J, Hróbjartsson A. The matching quality of experimental and control interventions in blinded pharmacological randomised clinical trials: a methodological systematic review. BMC Med Res Methodol. 2016;16:18. doi: 10.1186/s12874-016-0111-9.

5. Brown AB, Klerman JA. Independent evaluation: insights from public accounting. Eval Rev. 2012;36:186-219. 